\title{
Fast 2D-3D Point-Based Registration Using GPU-Based Preprocessing for Image-Guided Surgery
}

\author{
Helen Hong ${ }^{1, *}$, Kyehyun Kim², and Seongjin Park ${ }^{2}$ \\ ${ }^{1}$ Division of Multimedia Engineering, College of Information and Media, \\ Seoul Women's University, 126 Gongreung-dong, Nowon-gu, Seoul 139-774, Korea \\ hlhongaswu.ac.kr \\ ${ }^{2}$ School of Computer Science and Engineering, Seoul National University, \\ San 56-1 Shinlim-dong, Kwonak-gu, Seoul 151-742, Korea \\ \{iamkgh, sjpark\}@cglab.snu.ac.kr
}

\begin{abstract}
We propose a 2D-3D point-based registration method that provides fast and efficient alignment of X-ray fluoroscopy and CT images. Our method is divided into two procedures: pre-operative and intra-operative procedures. For pre-operative procedures, we generate digitally reconstructed radiographs (DRRs) from 3D volume using graphics hardware. In intra-operative procedures, we perform a hierarchical registration that includes in-plane registration using principal axes method and out-plane registration using minimal error searching method in spherical coordinates. This method reduces a degree of freedom from 6-DOF to 2-DOF. Experimental results using 2 cardiac phantoms show that our DRRs generation method is more than 150 times faster than software-based ray casting methods, and our hierarchical registration technique effectively matches DRRs and 2D images.
\end{abstract}

\section{Introduction}

Three-dimensional (3D) imaging modalities such as computed tomography (CT) and magnetic resonance (MR) imaging are widely used in clinical diagnosis and treatment planning due to their ability to produce detailed anatomical structures of human organs. However due to the manner in which the images are generated, both techniques have limitations in their use as a modality for guiding interventional procedures. For interventional procedures, images need to be produced in real-time and this is commonly done with ultrasound or X-ray fluoroscopy. The drawbacks of these modalities are that they fail to provide adequate spatial information. Combining the benefits of both types of imaging modalities could produce a system highly suitable for interventional procedures. Therefore, the registration of pre-operative $3 \mathrm{D}$ volume to intraoperative 2D images could provide complementary information for tracking the spatial location of medical instruments during image-guided surgery [1-4].

A major obstacle to this proposal is that images obtained with 3D scanning modalities have different dimensions from those obtained using 2D scanning modalities. To estimate geometrical transformation by bringing two modalities into spatial alignment, it

* Corresponding author. Seoul Women's University; Phone: 82-2-970-5756. 
is essential that two different images are compared in the same space[5]. There are two possible approaches to dealing with this $2 \mathrm{D}-3 \mathrm{D}$ registration.

- Convert the $2 \mathrm{D}$ image to a 3D image resulting in a 3D-3D registration

- Convert the $3 \mathrm{D}$ image to a $2 \mathrm{D}$ image resulting in a $2 \mathrm{D}-2 \mathrm{D}$ registration

From a practical point of view, 3D-3D registration, i.e. reconstructing 3D volume from $2 \mathrm{D}$ images requires numerous projection acquisitions and a large computation time[6]. It is consequently more feasible to simulate 2D images from $3 \mathrm{D}$ volume[5,7]. To do this, digitally reconstructed radiographs(DDRs) have to be first produced. These images are generated by a ray-casting algorithm that computes the amount of light that a virtual ray from an X-ray light source penetrates the $3 \mathrm{D}$ volume as shown in Fig. 1. Since this algorithm visits every voxel of 3D volume and computes while generating the projection image, it needs a large computation time. To reduce the computation time, software-based techniques have been proposed but are limited in their ability to generate simulated projection images in real-time.

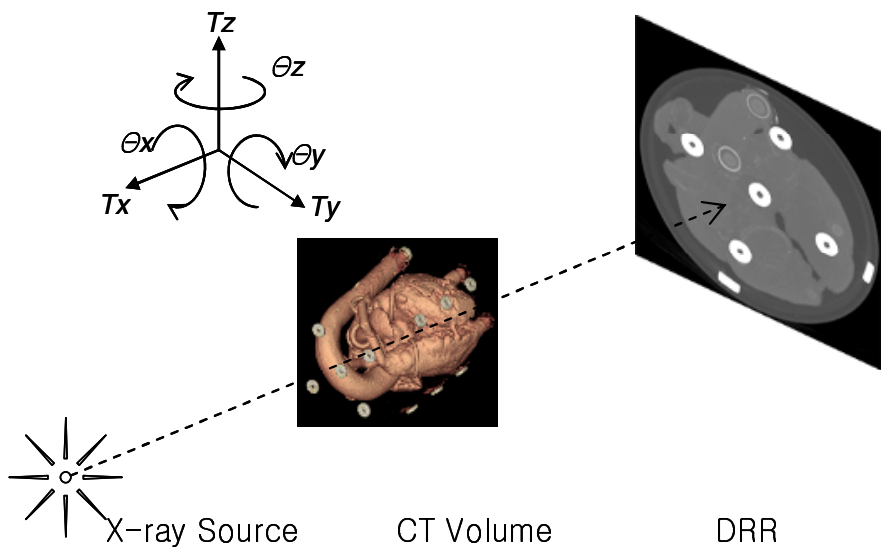

Fig. 1. The principle of DRRs generation from CT volume

In order to overcome this limitation, we propose a fast 2D-3D point-based registration using Graphics Processing Unit (GPU)-based preprocessing. Our main contribution is to accelerate DRRs generation using graphics hardware in pre-operative procedures and to perform hierarchical registration in intra-operative procedure that includes in-plane and out-plane registrations. Our hierarchical registration significantly increases the accuracy by reducing the degrees of freedom from 6 to 2 .

The organization of the paper is as follows. In Section 2, we discuss how to generate DRRs using graphics hardware. Then we propose a hierarchical registration to find correspondences between 2D X-ray fluoroscopy images and 3D CT volume. In Section 3, experimental results show how our methods accelerate the procedure for 2D-3D registration. This paper is concluded with brief discussion of the results in Section 4. 


\section{Fast 2D-3D Point-Based Registration}

For the registration of $3 \mathrm{D}$ volume and $2 \mathrm{D}$ images, we followed the pipeline shown in Fig. 2. The method is divided into two procedures: pre-operative and intra-operative procedures. For the pre-operative stage, DRRs are generated from 3D volumes using graphics hardware. For the intra-operative stage, hierarchical registration is performed by a technique that includes in-plane registration using a principal axes method and out-plane registration using a minimal error searching method in spherical space. In both stages, confirmation markers are automatically detected and segmented.

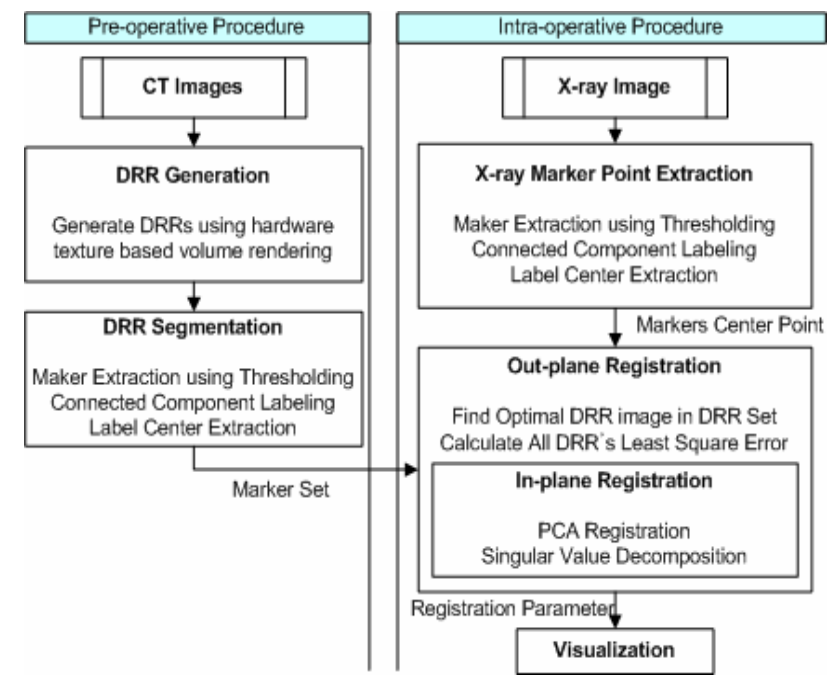

Fig. 2. The pipeline of proposed method using GPU-based preprocessing for Image-Guided Surgery

\subsection{The Generation of DRRs Using Graphics Hardware}

The DRRs are generated by texture-based volume rendering using hig-level shading language. Generation is composed of three steps. Firstly the 3D volume is changed from 12 bit to 8 bit. Secondly the proxy geometry is generated using perspective projection. Finally the compositing is performed using maximum intensity projection.

The reason for changing the 3D volume from 12 bit to 8 bit is that current graphics hardware has a limitation in loading 3D volume to three-dimensional texture memory in GPU when the resolution of the 3D volume is over 512 × 512 × 512 bits. The main purpose of our method is to align confirmation markers between DRRs projected from 3D volume and 2D images. In addition, the confirmation markers have a high density values. Thus, it is possible to use higher 8 bit from 12 bit in $3 \mathrm{D}$ volume. In general, proxy geometry is generated using parallel projection. In order to simulate X-ray images, we have generated proxy geometry using the technique of perspective 
projection as shown in Fig. 3. We map three-dimensional texture memory in GPU to the proxy geometry. The mapped slices onto the proxy geometry render using compositing modes that include maximum intensity projection, average intensity projection and minimum intensity projection.

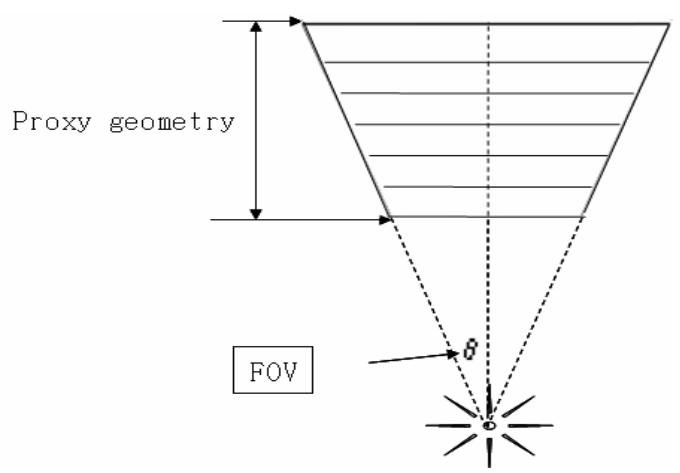

Fig. 3. A generation of proxy geometry using perspective projection

\subsection{Automatic Confirmation Marker Segmentation}

For point-based registration, confirmation markers are needed to automatically detect and segment. Our method is composed of three steps. Firstly, candidate regions of confirmation markers in each image are segmented by using thresholding [8]. The pixels above 2300 Hounsefield units are considered as the candidate of confirmation markers. Then these candidate regions are saved as binary images as shown in Fig. 4(a). Secondly, noise or other features in the binary images are removed by using connected component labeling. For this, candidate regions of confirmation marker shown in Fig. 4(b) are compared with standard confirmation marker model. If candidate regions of confirmation markers are smaller or larger than the standard confirmation marker model, they are considered as noise or other features and are removed. Finally, centroids of extracted confirmation markers are computed.

\subsection{Hierarchical Registration}

The purpose of $2 \mathrm{D}-3 \mathrm{D}$ registration is to align the $3 \mathrm{D}$ volume obtained from preoperative imaging modalities with 2D images obtained from intra-operative imaging modalities. In order to determine geometrical transformations in three dimensions, translations and rotations in $\mathrm{x}-, \mathrm{y}-$, and $\mathrm{z}$-axis are computed with six degree-offreedom (DOF). However, this approach dramatically increases the number of computations. To address this problem we propose a hierarchical registration that includes in-plane registration and out-plane registration. This method reduces the computation requirement from $6 \mathrm{DOF}$ to $2 \mathrm{DOF}$. 


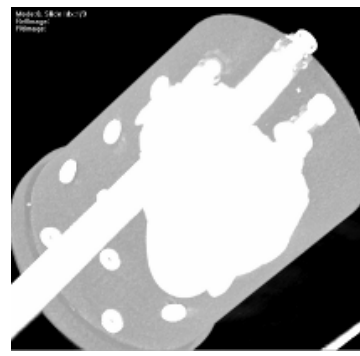

(a)

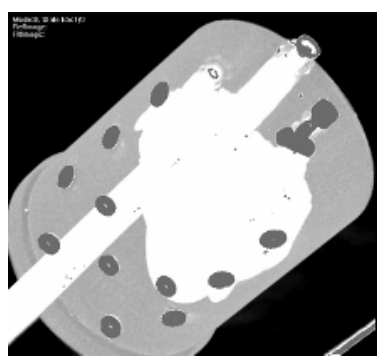

(b)

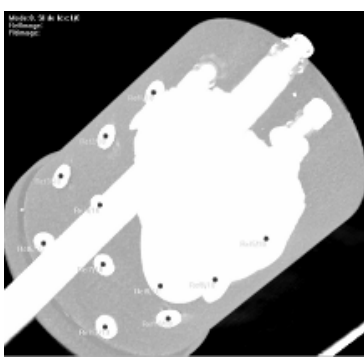

(c)

Fig. 4. A concept of automatic marker detection and segmentation (a) DRR image (b) candidate regions (c) noise removal and centroid extraction

For in-plane registration, we determine optimal translation and rotation vectors of confirmation markers using the principle axes method. Each axis of DRRs and 2D images are computed using Singular Value Decomposition (SVD) and are used to align these two images[9,10]. SVD is defined as Eq. 1. The in-plane rotation vector $\theta$ is the differential angle between axes. The in-plane translation vectors, $T_{X}, T_{Y}$ for each axes is computed by the weighted mean of the markers' center positions.

$$
\begin{gathered}
A=U \Sigma V^{T} \Leftrightarrow \quad \Leftrightarrow=U^{T} A V \\
\Sigma=\operatorname{diag}\left(\sigma_{1}, \cdots, \sigma_{r}\right)
\end{gathered}
$$

where $\mathrm{U}$ and $\mathrm{V}$ are unitary, and $\Sigma$ is real diagonal elements. $\sigma_{\mathrm{i}}$ is called the singular values.

Out-plane registration is used to compute the position of the source of $2 \mathrm{D}$ images in $3 \mathrm{D}$ space. To find the source position, we apply two rotation vectors of spherical coordinate systems and search the rotation vectors that optimize the correspondence between $3 \mathrm{D}$ volume and 2D images. In order to estimate the similarities, we use the root-mean-squared (RMS) error of the result of in-plane registration like Eq. 2.

$$
R M S E=\sqrt{\frac{1}{n} \sum_{j=1}^{n}\left\|P_{i j}-T_{j}\right\|^{2}}
$$

where $T_{i}$ is x-ray marker and $P_{i j}$ is nearest marker of CT with $T_{i}$.

\section{Experimental Result}

All our implementation and tests were performed using a personal computer (PC) equipped with an Intel Pentium 4, 2.4 GHz CPU and 1GB memory. The graphics hardware was ATI Radeon 9600 GPU with 256 MB of memory. Our method was applied to two cardiac phantom datasets to evaluate its accuracy and computation time. 
Table 1 shows the datasets derived from the 2 cardiac phantoms used for the study. Three groups of datasets were used:

- Dataset A and B had 3600 DRRs taken at intervals of 0.5 degree

- Dataset C and D had 900 DRRs taken at intervals of 1.0 degree

- Dataset E and F had 225 DRRs taken at 2.0 degree interval

Table 1. Image conditions of experimental datasets

\begin{tabular}{cccccc}
\hline Dataset & CT dataset & $\begin{array}{c}\text { Image } \\
\text { resolution }\end{array}$ & Slice \# & $\begin{array}{c}\text { DRR } \\
\text { Interval }\end{array}$ & $\begin{array}{c}\text { Slice \# } \\
\text { (DRR) }\end{array}$ \\
\hline \hline A & CT 1 & $512 \times 512$ & 566 & 0.5 & 3600 \\
\hline B & CT 2 & $512 \times 512$ & 391 & 0.5 & 3600 \\
\hline C & CT 1 & $512 \times 512$ & 566 & 1.0 & 900 \\
\hline D & CT 2 & $512 \times 512$ & 391 & 1.0 & 900 \\
\hline E & CT 1 & $512 \times 512$ & 566 & 2.0 & 225 \\
\hline F & CT 2 & $512 \times 512$ & 391 & 2.0 & 225 \\
\hline
\end{tabular}

We have compared our technique with software-based ray casting. This is an image order algorithm used in computer graphics to render three-dimensional scenes to twodimensional scenes by following rays of light from the eye of the observer to a light source. Table 2 shows a comparison of the DRRs generation time using the 2 different techniques. Our method is over 150 times faster than software-based ray-casting.

Fig. 5 shows the results obtained by generating DRRs using our proposed method based on graphics hardware in comparison with software-based ray casting. It indicates that the image quality of DRRs generated from our method is as good as that of software-based ray-casting.

Fig. 6 shows the result of segmentation of confirmation markers in DRRs. The background image is X-ray fluoroscopy with circles indicating confirmation markers in X-ray fluoroscopy, and crosses indicating confirmation markers in DRRs.

Fig. 7 shows the result of registration of DRRs generated from the 3D cardiac phantoms and X-ray fluoroscopy. In the case of registration of 2-degree interval DRRs, the confirmation markers of the DRRs are not aligned with those of the X-ray fluoroscopy. However, with registration of 1 or 0.5 degree interval DRRs, the confirmation markers are almost perfectly matched.

Table 2. The comparison of DRRs generation time

\begin{tabular}{ccc}
\hline Dataset & $\begin{array}{c}\text { Proposed graphics } \\
\text { hardware method (min) }\end{array}$ & $\begin{array}{c}\text { Software-based ray-casting } \\
\text { method (min) }\end{array}$ \\
\hline \hline A & 24.21 & 5707.85 \\
B & 23.48 & 3664.28 \\
C & 6.02 & 1427.01 \\
D & 5.87 & 916.06 \\
E & 1.50 & 356.67 \\
F & 1.47 & 229.03 \\
\hline
\end{tabular}




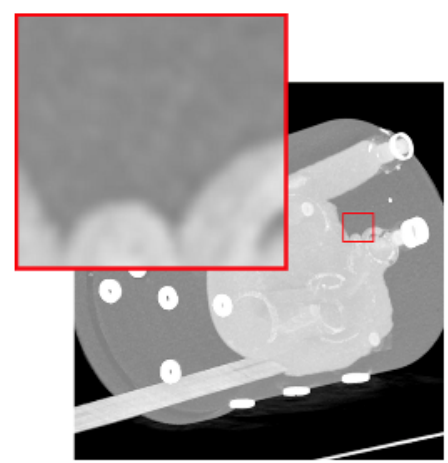

(a)

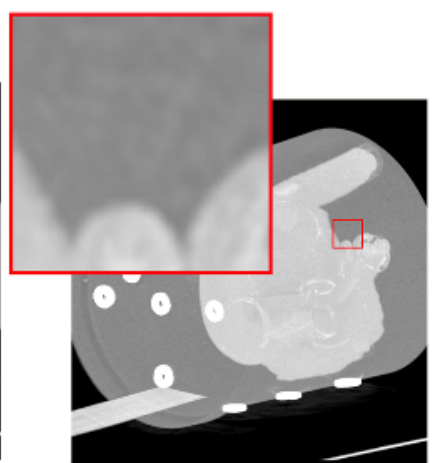

(b)

Fig. 5. The results of DRRs generation (a) proposed graphics hardware-based method (b) software-based ray casting

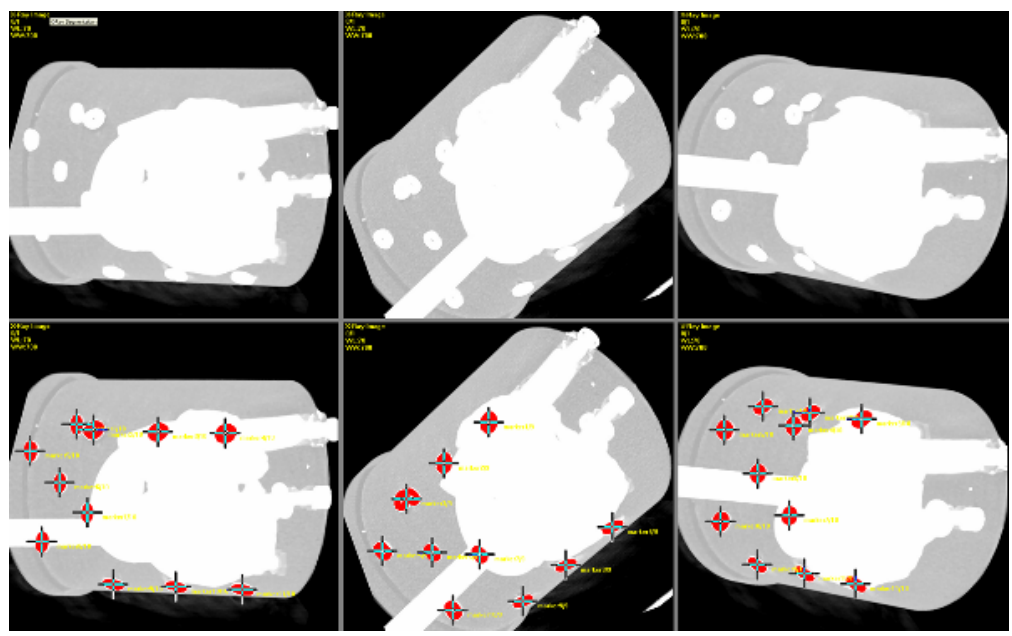

Fig. 6. The results of segmentation of confirmation markers in X-ray fluoroscopy and DRRs

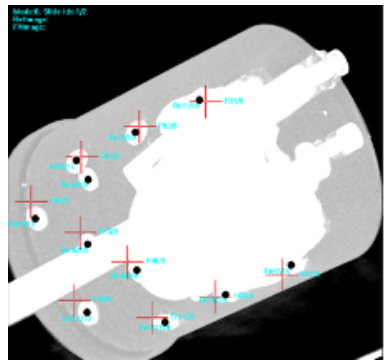

(a)

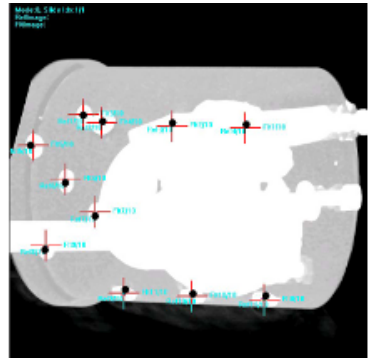

(b)

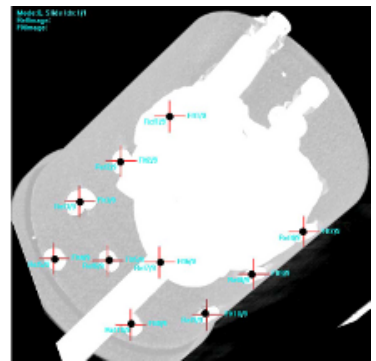

(c)

Fig. 7. The results of 2D-3D point-based registration with (a) registration of 2.0 degree interval DRRs, (b) registration of 1.0 degree interval DRRs, (c) registration of 0.5 degree interval DRRs 
To further evaluate the accuracy of the method, we studied the root mean square error between the confirmation markers of DRRs and X-ray fluoroscopy (a distance referred to as the ground truth). If both markers are identically aligned there will be no distance or angulation between them. Our results demonstrated that the distance between the 2 markers varied from 12 to $47 \mathrm{~mm}$ and the rotation between them varied from 31.6 to 56.5 degrees in all 3 axes (see Table 3 ). The root mean square error varied between 0.7 and $2.3 \mathrm{~mm}$ for distance and 0.5 and 2.8 for angulation.

Table 3. The accuracy evaluation using RMS error

\begin{tabular}{|c|c|c|c|c|c|c|}
\hline \multirow{3}{*}{ Dataset } & \multicolumn{2}{|c|}{ Translation } & \multicolumn{3}{|c|}{ Rotation } & \multirow{3}{*}{$\begin{array}{c}\text { RMSE } \\
(\mathrm{mm})\end{array}$} \\
\hline & \multicolumn{2}{|c|}{ Ground-Truth Parameters } & \multicolumn{3}{|c|}{ Ground-Truth Parameters } & \\
\hline & $\left(\mathrm{T}_{\mathrm{x}}, \mathrm{mm}\right)$ & $\left(\mathrm{T}_{\mathrm{y}}, \mathrm{mm}\right)$ & $(\theta x, \mathrm{deg})$ & $(\theta y, \operatorname{deg})$ & $(\theta z, \operatorname{deg})$ & \\
\hline \multirow{2}{*}{ Data A } & 30.9 & 30.1 & 53.5 & 48.0 & 56.5 & \multirow{2}{*}{2.7} \\
\hline & 31.0 & 29.0 & 53.7 & 47.9 & 56.3 & \\
\hline \multirow{2}{*}{ Data B } & 12.3 & 17.6 & 35.5 & 50.5 & 45.5 & \multirow{2}{*}{1.8} \\
\hline & 12.0 & 18.0 & 35.7 & 50.3 & 45.4 & \\
\hline \multirow{2}{*}{ Data C } & 29.7 & 46.4 & 32.0 & 50.0 & 42.0 & \multirow{2}{*}{11.3} \\
\hline & 29.0 & 47.0 & 31.6 & 50.5 & 41.6 & \\
\hline \multirow{2}{*}{ Data D } & 22.5 & 30.8 & 36.0 & 42.0 & 42.0 & \multirow{2}{*}{8.6} \\
\hline & 24.0 & 30.0 & 36.2 & 41.6 & 41.9 & \\
\hline \multirow{2}{*}{ Data E } & 20.6 & 28.8 & 54.0 & 54.0 & 48.0 & \multirow{2}{*}{19.7} \\
\hline & 21.0 & 27.0 & 54.9 & 53.5 & 49.4 & \\
\hline \multirow{2}{*}{ Data F } & 15.7 & 15.8 & 47.0 & 36.0 & 54.0 & \multirow{2}{*}{22.7} \\
\hline & 16.0 & 15.0 & 46.1 & 36.9 & 54.8 & \\
\hline
\end{tabular}

\section{Conclusion}

We have developed a novel technique of 2D-3D registration of three-dimensional CT dataset and X-ray fluoroscopy. In pre-operative procedure, the DRRs generation method based on graphics hardware was performed rapidly. The automatic confirmation marker segmentation could remove noise and other features in both DRRs and Xray fluoroscopy. The hierarchical registration including in-plane and out-plane registrations could reduce the search space from 6 DOF to 2 DOF. In our experiments, we use 3D cardiac phantom dataset to evaluate accuracy and computation time. The image quality of DRRs generated from our method is as good as that of software-based ray casting. In addition, the DRRs generation using graphics hardware is over 150 times faster than software-based ray casting. Experimental results showed that our DRRs generation method performs very fast and the hierarchical registration effectively matches the DRRs and 2D images.

\section{Acknowledgement}

This study is supported by Bahrom research fund from Seoul Women's University, 2006. 


\section{References}

1. Gage, B.F., Waterman, A.D., Shannon, W., Boechler, M., Rich, M.W., Radford, M.J., Validation of clinical classification schemes for predicting stroke: results from the National Registry of Atrial Fibrillation, Jama, Vol. 285 (2001) 2864-2870.

2. Cox, J.L., Shuessler, R.B., D'Agostino H.J., Jr., et al., The surgical treatment of atrial fibrillation. III. Development of a definitive surgical procedure, J. Thorac Cardiovasc Surg, Vol. 101 (1991) 569-583.

3. Wyse, D.G., Waldo, A.L., DiMArco, J.P. et al., A comparison of rate control and rhythm control in patients with atrial fibrillation, N Engl J Med, Vol. 347 (2002) 1825-1833.

4. Swartz, J., Perrersels, G., Silvers, J., Patten, L., Cervantez, D., A catheter based curative approach to atrial fibrillation in humans, Circulation (1994).

5. Zollei, E., Grimson, A., Norbash, W. Well., 2D-3D rigid registration of X-ray fluoroscopy and $\mathrm{CT}$ images using mutual information and sparsely sampled histogram estimators, IEEE CVPR (2001).

6. Berthold, K.P., Horn, Closed-form solution of absolute orientation using unit quaternions, Journal of the Optical Society of America, Vol. 4, No. 4 (1987) 629-642.

7. J. Weese, R. Gocke, G.P. Penny, P. Desmedt, T.M. Buzug, H. Schumann, Fast voxelbased 2D-3D registration algorithm using a volume rendering method based on shear-warp factorization, Proc. of SPIE Medical Imaging, Vol. 3661 (1999) 802-810.

8. R.G. Gonzalez, R.E. Woods, Digital Image Processing, Addison-Wesley (1993) 443-458.

9. Rusinek, H., Tsui, W., Levy, A.V., Noz, M.E., and de Leon, M.J., Principal axes and surface fitting methods for three-dimensional image registration, J. Nucl. Med., Vol. 34 (1993) 2019-2024.

10. Alpert, N.M., Bradshaw, J.F., Kennedy, D., Correia, J.A., The principal axis transformation - a method for image registration, J. Nucl. Med., Vol. 31 (1990) 1717-1722. 